\title{
MULTI-SCALE SIMULATION OF SPHERICAL AGGREGATES
}

\section{ARnAUd DELARUE AND DOMINIQUE JEULIN}

Centre de Morphologie Mathématique, Ecole des Mines de Paris, 35 rue Saint Honoré 77300 Fontainebleau, France e-mail: delarue@cmm.ensmp.fr (Accepted October 8, 2001)

\begin{abstract}
This paper presents a method to simulate high density non overlapping sphere structures with anisotropy and heterogeneity.
\end{abstract}

Keywords: 3D mathematical morphology, non-overlapping spheres, structure simulation.

\section{INTRODUCTION}

Morphological models of random sets (Matheron, 1975; Serra, 1982) provide a means to represent in a synthetic way the morphological properties of microstructures, and to generate 3D simulations using the identification of models based on the available information, namely part of the Choquet capacity.

The aim of this work is to propose multiscale simulations of anisotropic structures containing aggregates of non-overlapping spheres. Such microstructures are very common in materials, and in the present case were studied in a joint research project (Delarue, 2000). Composite materials were elaborated from an aluminium or a PMMA matrix incorporating Zircon or Zircon-Silica spherical inclusions. Materials were prepared in the GEMPPM (INSA de Lyon) and in the LTPCM (Grenoble). 3D images of this materials are obtained by X-ray microtomography in the Synchrotron of the ESRF (Grenoble) with a $2 \mu \mathrm{m}$ resolution per voxel.

In the Centre de Morphologie Mathématique, we characterized these structures by 3D measurements such as the covariance in various directions, a tortuosity index obtained from 3D geodesic propagation, local volume fraction of inclusions, distance functions and angular distributions (to study the anisotropy of the neighborhood of inclusions) (Delarue, 2000).

The first part of this paper is a presentation of a standard method to generate random homogeneous and isotropic non-overlapping sphere structure, and problems related to high densities. The second part presents measurements, able to characterize spatial distribution from multi scale histograms. The next part presents the results of the measurements on 3D microtomography images of real materials. The last part describes an algorithm of multi-scale simulations, to obtain heterogeous and anisotropic hard spheres structures with a high density.

\section{D HIGH DENSITY HARD SPHERE SIMULATION}

The implantation of non-overlapping spheres with random coordinates is practically impossible if the density is larger than 30 or $35 \%$. To generate simulations with a higher density of hard spheres, it is better to start from an ordered dense structure, like cfc (cubic face centered). But this kind of structure is completely ordered. Disorder can be introduced by deleting some spheres at random and by random translation of the remaining spheres.
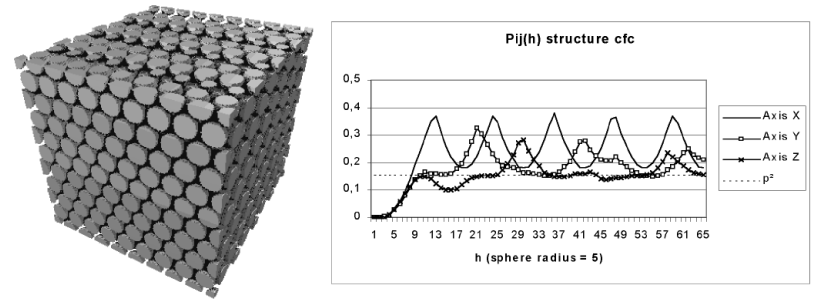

Fig. 1. Image and 2 points correlation function $P_{i j}(h)=P\left\{x \in A_{i} ; x+h \in A_{j} ; i \neq j\right\}$ of a cfc $3 D$ structure (40\%). 


\section{ALGORITHM OF SIMULATION}

In this part, we consider simulation of hard spheres with a fixed radius $R=1$. The first step of a random hard sphere structure simulation with a high density is to implant ordered hard sphere with cfc order and with the higher hard core radius $R_{H c}$. The maximum theoretical hard core radius, for a $p$ density structure, is given by:

$$
R_{H c}=\sqrt[3]{\frac{\pi}{3 \sqrt{2} p}}
$$

The highest density, when $R_{H c}=1$, is $74.04 \%$. This result corresponds to Kepler's conjuncture, which was recently proved by Th. Hales (Aste, 2000).

After implantation, spheres are moved by random translations. At each step, a sphere and a direction is chosen. The translation is fifty times smaller than the sphere radius. This is accepted if the translation of the sphere is made without any sphere overlap. If it is accepted, the same translations are repeated 10 times. This method is very fast but it is important to know how many translations are needed, and to define a criterion of convergence of the process.

\section{CONVERGENCE}

Measurement of the probability for two points to be in two separated spheres $P\left\{x \in A_{i} ; x+h \in\right.$ $\left.A_{j} ; i \neq j\right\}$ is used to characterize the spherical packing. For a cfc structure like that in Fig. 1, this measurement produces curves with many oscillations. After translations of spheres, the order disappears in the structure and oscillations decrease (Fig. 2). The convergence criterion $C_{c}$ is defined by the following formula:

$$
C_{c}=\int_{h=2 r}^{10 r}(10 r-h)\left(P\left\{x \in A_{i} ; x+h \in A_{j} ; i \neq j\right\}-p^{2}\right)^{2} d h .
$$

For a low density (20 or $30 \%)$, few translations are needed for convergence. For a higher density (40 or $50 \%$ ) the number of translations increases quickly (Fig. 3). The highest volume concentration of random spheres simulated by this technique is close to $55 \%$.

\begin{tabular}{|r|l|}
\hline $\begin{array}{l}\text { Density of hard } \\
\text { spheres }\end{array}$ & $\begin{array}{l}\text { Number of translations by } \\
\text { sphere }\end{array}$ \\
\hline $20 \%$ & 120 \\
\hline $30 \%$ & 250 \\
\hline $40 \%$ & 700 \\
\hline $50 \%$ & 2700 \\
\hline
\end{tabular}
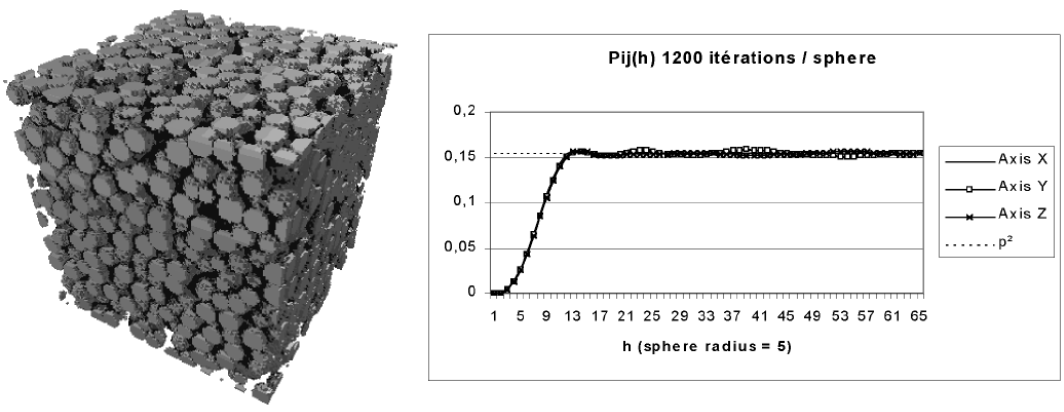

Fig. 2. Image and 2 points correlation function of $3 D$ random hard sphere structure (40\%).
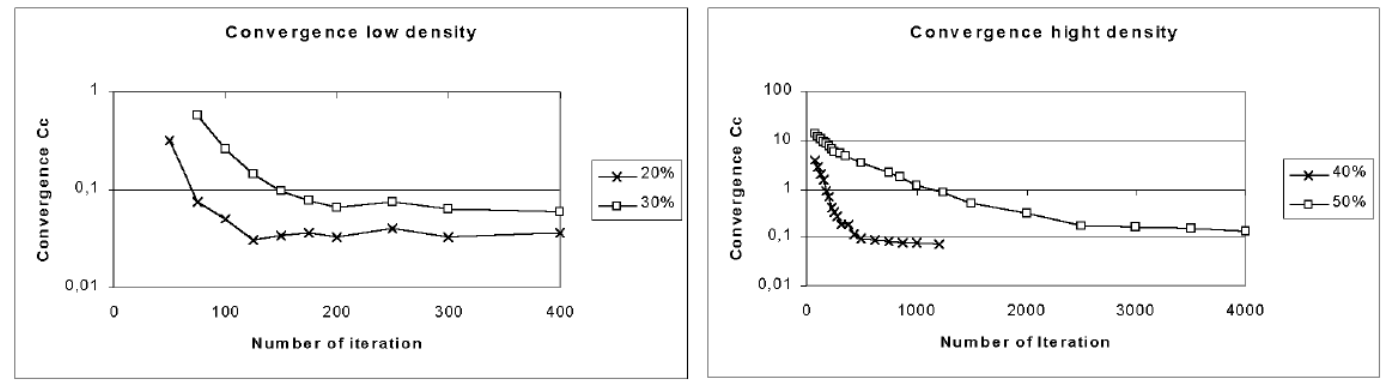

Fig. 3. Convergence test: the number of iterations by sphere increases with the density. 


\section{CHARACTERIZATION OF THE SPATIAL DISTRIBUTION FROM MULTI SCALE HISTOGRAMS}

In this part, we present a 3D characterization of granular arrangements based on the estimation of the histogram of the number of spheres included in a characteristic volume. To account for the anisotropy of the structure, this volume is a parallelepiped with different orientations. Different sizes of parallelepiped are used to obtain information at various scales.

In Fig. 4, histograms of local grain numbers are the same in both directions $x$ and $y$. But, if a longer parallelepiped is taken (Fig. 5), histograms become anisotropic. In this last histogram it is possible to find the main direction of aggregates (in this case, the horizontal direction). With parallelepipeds showing different orientations, it is possible to study the size and orientation of aggregates.
This measurement is quite fast because, for example, for an image of $1.2 \times 1.2 \times 1.2 \mathrm{~mm}^{3}$, with a resolution of $2 \mu \mathrm{m}$ and measurements with parallelepipeds with dimensions $64 \times 64 \times 128 \mu^{3}$ and $64 \times 64 \times 256 \mu^{3}$ every $32 \mu \mathrm{m}$ returns a measurement more than $10^{3}$ faster than covariance. The main advantages of this type of measurements are the characterization of heterogeneity and anisotropy, and the very low cost of calculation.

\section{MEASUREMENT ON REAL MATERIALS}

Figs. 6 and 7 show measurements obtained on two different structures (Delarue, 2000). The first structure (Fig. 6) is homogeneous and isotropic without aggregates, with $40 \%$ volume fraction of spheres.
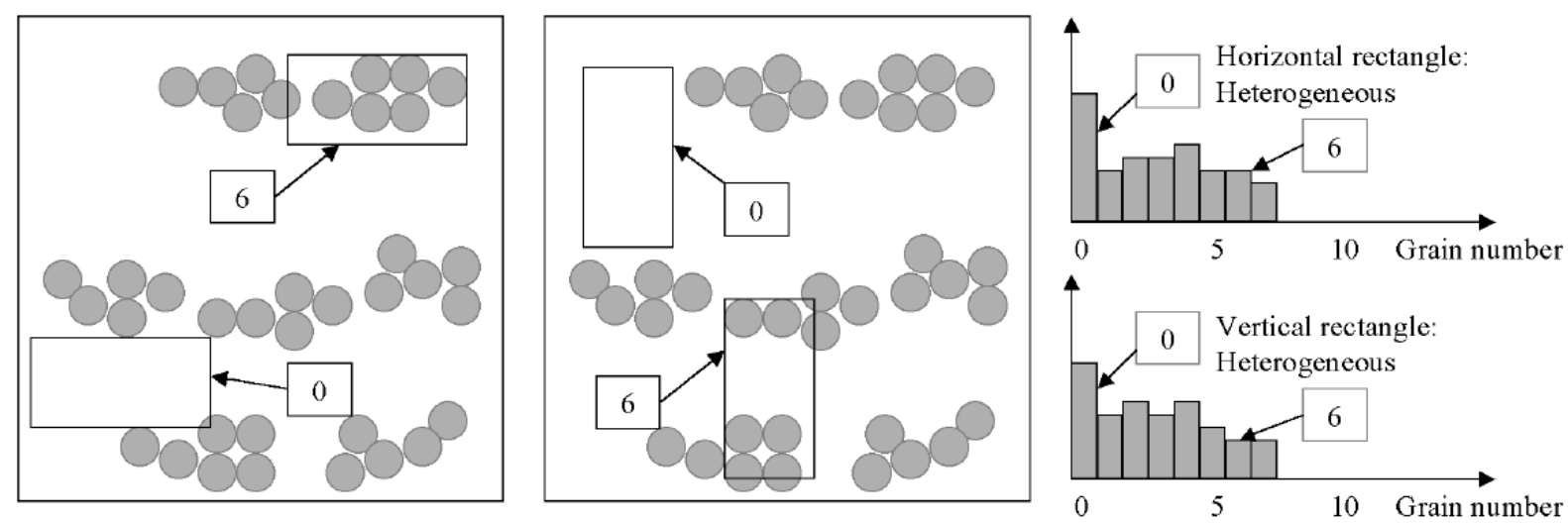

Fig. 4. Histogram of local grain number. At this scale, the structure is isotropic.
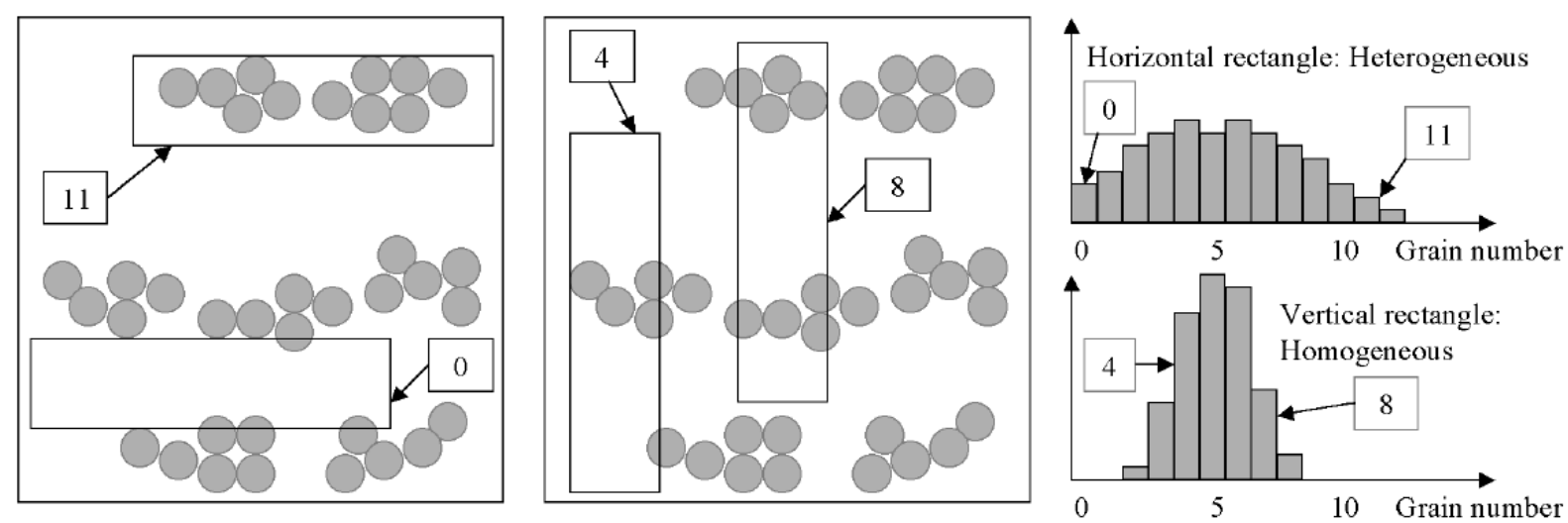

Fig. 5. Histogram of local grain number. At this scale, the structure is anisotropic. 


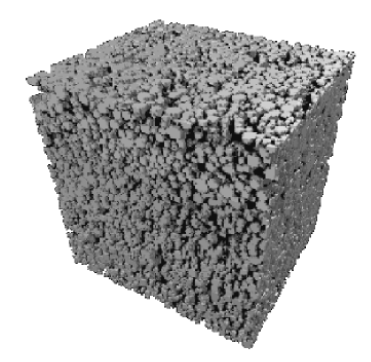

PMMA- $\mathrm{ZrO}_{2}-$ Silicon (40\%), $1,2 \times 1,2 \times 1,2 \mathrm{~mm}^{3}$, Sphere about 25 microns radius and histogram of local grain number with 2 sizes of parallelepipeds.
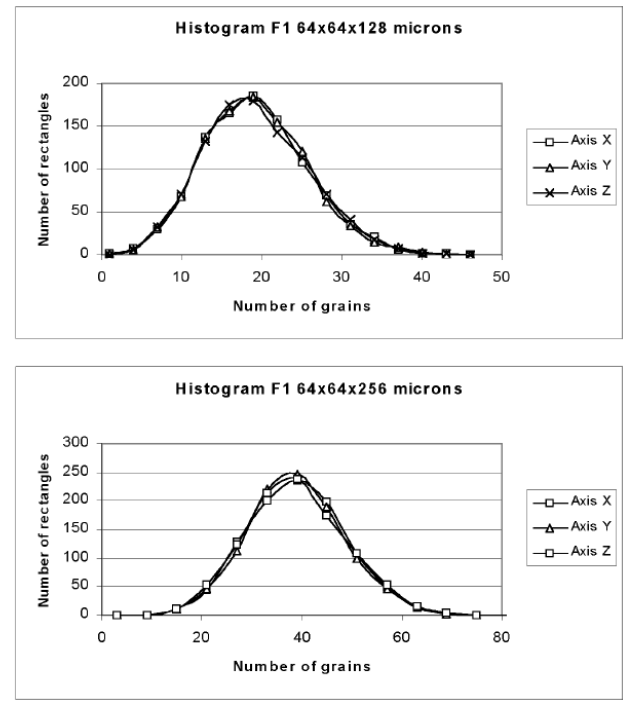

Fig. 6. Measurement on an homogeneous and isotropic structure.

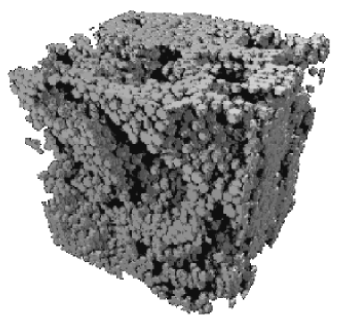

$\mathrm{Al}-\mathrm{ZrO}_{2}(35 \%)$, $1,0 \times 1,0 \times 1,0 \mathrm{~mm}^{3}$, Sphere about 25 microns radius and histogram of local grain number with 2 sizes of parallelepipeds.

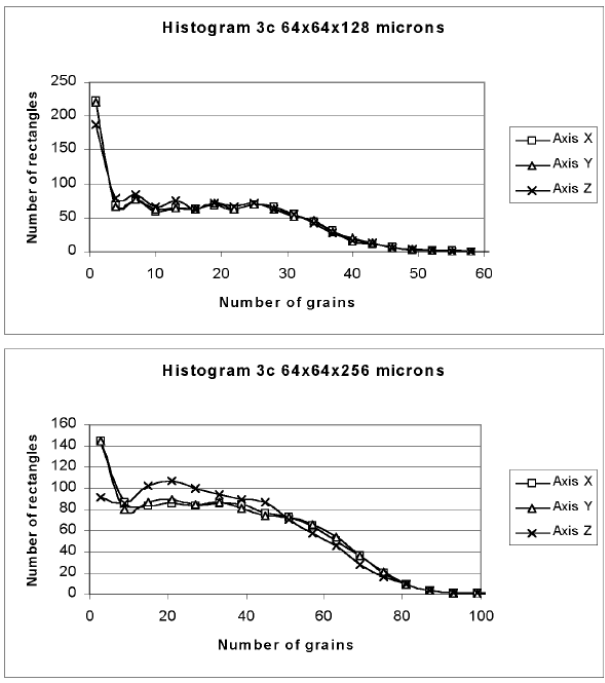

Fig. 7. Measurement on anisotropic aggregates.

The second structure (Fig. 7) is a $35 \%$ aggregate structure which was compressed along the $z$ axis. The heterogeneity is apparent for the two types of histograms. The first one is isotropic and corresponds to the smaller dimension of aggregates. The histogram with the larger parallelepiped is sensitive to the larger dimension of the aggregate. As the covariance, this measurement can detect that aggregates are flat (like disks orthogonal to the $\mathrm{Z}$ axis).

Real structures need two types of information to be completely characterized. The characterization of the spatial localization of inclusions obtained with the measurements presented here, and the granulometry because of the non unique hard sphere radius of inclusions. The granulometry can be obtained by labelisation of inclusions (a small erosion is sometimes required to separate grains) and volume measurement of each of them. We assume that inclusions are spherical. Fig. 8 presents the granulometry of a homogenous and of a heterogenous structure.

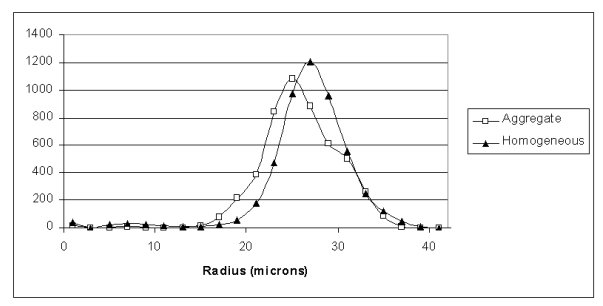

Fig. 8. Granulometry of real materials (distribution of volumes of inclusions).

\section{STRUCTURE SIMULATION}

Starting from the experimental histograms of local grain numbers and the granulometry, shown to be characteristic of a given material, it is possible to 
simulate three dimensional structures following the same distributions. In a first step, a hard sphere structure with a random distribution (for a high density in most studied materials) is simulated. Then random translations of the spheres are generated, controlling the difference between the histograms of local sphere numbers of the reference and of simulated structures. The size of the translation decreases when the simulation proceeds, like cooling in a thermodynamical system. If $\mathrm{N}$ is the total number of translations, for the i-th translation, the maximal sphere range $d(i)$ is given by the formula,

$$
d(i)=d_{0} e^{-k \frac{i}{N}} .
$$

Sphere motion is accepted if this difference decreases. The procedure is iterated until a given number of steps (typically $N=25000$ for about 1000 spheres) or a level of error is reached. As the histogram is estimated for each sphere movement, the importance of a very fast measurement process is clear. Such constrained simulations follow a simulated annealing process (Yeong, 1998; Manwart, 2000). Similar approaches were followed in 2D: simulations reproduce the histograms distributions of the number of pixels of the microstructure in different windows (Srivastava, 1994), or the covariance of non overlapping discs (Rintoul, 1997).

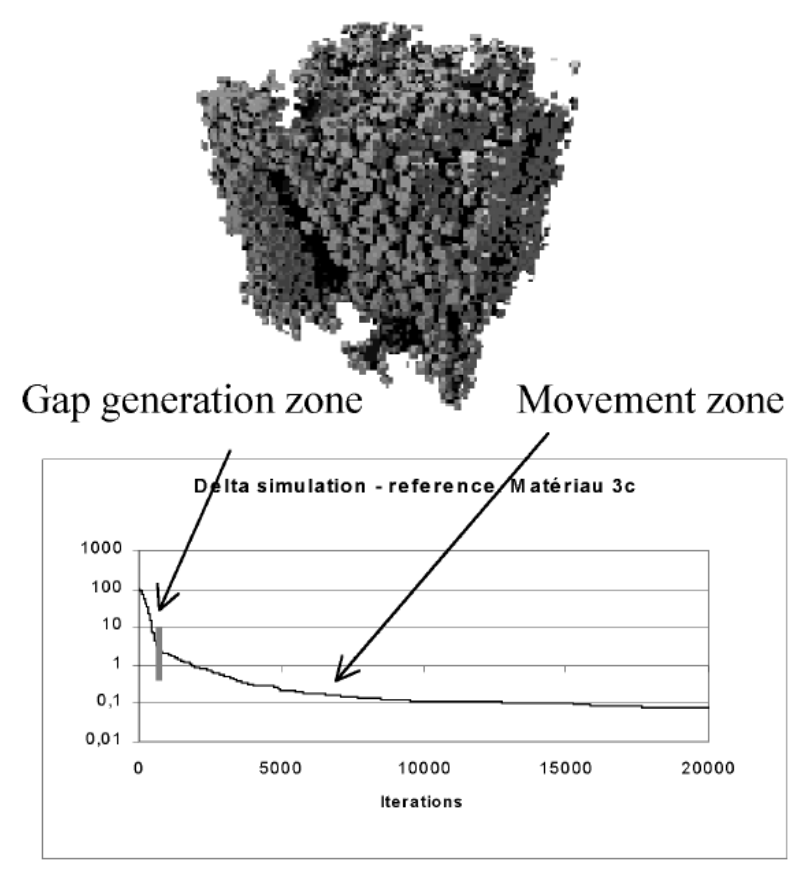

\section{GAP GENERATION}

Structures with a high heterogeneity and a high density are very difficult to simulate by this method. Random translations (under histogram control) of individual spheres can hardly generate aggregates with a high local density and large empty spaces between grains. A solution to this problem was developed by the controlled generation of gaps (Fig. 9).

To simulate a heterogeneous structure with the density reference $D_{\text {ref }}$, a homogeneous hard sphere structure is generated with the density $D_{\text {Simul }}\left(D_{\text {Simul }}>\right.$ $D_{\text {ref }}$ ). After that, spheres are deleted and the difference between the histograms of local sphere number of reference and simulated structure is controlled. If the difference decreases, the deletion is accepted. When the density of the simulated structure $D_{\text {Simul }}$ reaches $D_{\text {ref }}$, random translations under the control of histogram differences start, as previously.

\section{RESULTS}

After simulation, structures are validated with respect to reference structures by a direct comparison of the histograms (to locate and explain their differences) and by estimating the probability for two points to be in two different inclusions, which is typical of the anisotropy of the distribution of inclusions (Delarue, 2000).
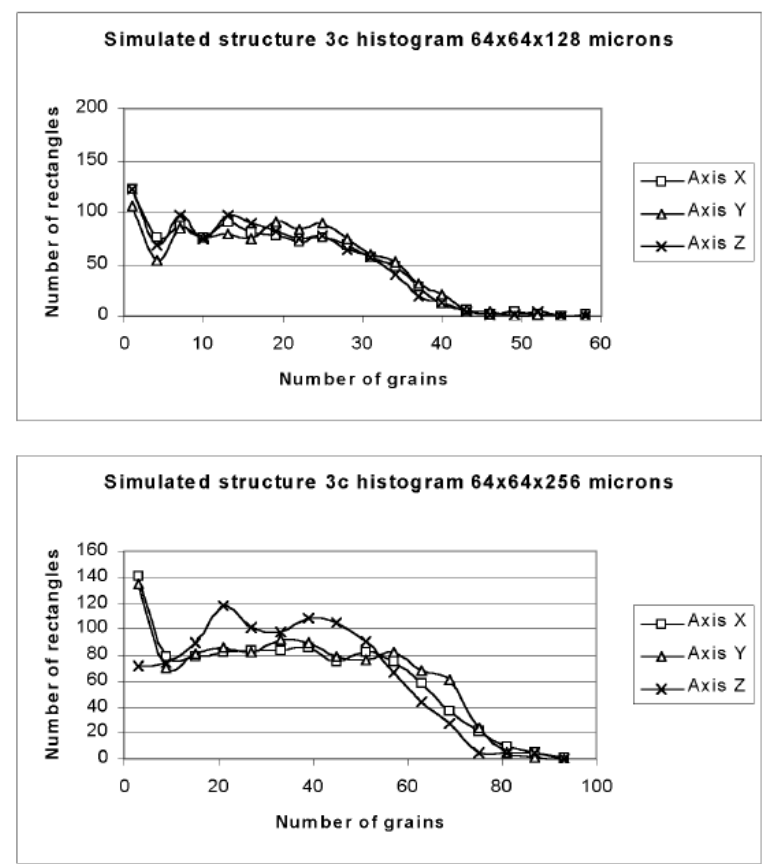

Fig. 9. Simulated structure. Image and measurement look similar to those in Fig. 7. 


\section{CONCLUSION}

In this paper we present the different steps to obtain hard sphere simulations using histograms of sphere center numbers included in a characteristic volume. For a homogeneous low density structure, only two steps are needed to obtain simulations: random implantation and random translation under a reference structure histogram condition. For higher densities, random implantation must be replaced by a dense periodic implantation and random translations. We show that the number of translations needed to converge to disorder increases with the density. In the case of very heterogeneous structures, four steps are needed: ordered implantation of spheres with a higher density than the reference one, random translation, random suppression and translation under control of the reference structure histograms.

\section{ACKNOWLEDGEMENTS}

This study was supported by CNRS (project 104 . Programme matériaux). A. Delarue is indebted to CNRS and to DGA for a grant.

\section{REFERENCES}

Aste T, Weaire D (2000). The pursuit of perfect packing. Bristol: Institute of Physics Publishing.
Delarue A, Jeulin D (2000). Morphologie 3D de Composite Granulaires, N-29/00/MM. Caractérisation Morphologique 3D de Matériaux Hétérogènes par Microtomographie X et Traitement d'image pour la prévision de Comportement Mécanique. Programme Matériaux du CNRS, Thème 12a, Projet N 104.

Matheron G (1975). Random sets and integral geometry. New York: J. Wiley.

Manwart C, Torquato S, Hilfer R (2000). Stochastic reconstruction of sandstones. Phys Rev E 62:893-9.

Rintoul MD, Torquato S (1977). Reconstruction of the structure of dispersions. J Colloid Interf Sci 186:46776.

Serra J (1982). Image analysis and mathematical morphology. London: Academic Press.

Srivastava RM (1994). An annealing procedure for honouring change of support statistics in conditional simulations. In: Dimitrakopoulos R, ed. Geostatistics for the next century. Dordrecht: Kluwer Academic Publishers, 277-90.

Yeong CLY, Torquato S (1998). Reconstructing Random Media: II. Three-Dimensional from Two-Dimensional Cuts. Phys Rev E 58:224-33. 